Classification

Physics Abstracts

$61.25 \mathrm{H}-82.70-05.90$

\title{
Further evidence of liquid-like correlations in polyelectrolyte solutions
}

\author{
Isabelle Morfin, Wayne F. Reed (**), Marguerite Rinaudo and Redouane Borsali (*) \\ CERMAV-CNRS and University Joseph Fourier, P.O. Box 53, 38041 Grenoble Cedex 9, France
}

(Received 29 June 1993, revised 21 February 1994, accepted 3 March 1994)

\begin{abstract}
Elastic, quasi elastic light scattering and viscosity experiments were used to investigate polyelectrolytic polysaccharide succinoglycan solutions at low solute concentration $C_{\mathrm{p}}$, and salt concentration $C_{\mathrm{a}}$. The highest degree of « organization » in the solution necessary to describe the observations is a simple liquid type correlation, manifested by broad angular static and dynamic scattering maxima of visible light for solutions at very low ionic strength. Letting the solutions stand undisturbed for a few days did not lead to a sharpenıng of the broad maxima, nor did lowering the temperature. The positions of these maxima scale roughly as $C_{\mathrm{p}}^{1 / 2}$. By adding salt, the maxima were found to maintain roughly the same position. The reciprocal diffusion coefficient $D^{-1}(q)$ corresponding to the liquid-like correlation state followed the intensity maxima, as has often been demonstrated for similar systems. No «slow mode» or « extraordinary regime» of diffusion was found associated with the static and dynamic light scattering maxima although extreme care in filtration of solution was necessary to avoid a spurious slow diffusional mode due to aggregates.
\end{abstract}

\section{Introduction.}

The solution properties of polyelectrolytes in media of different ionic strength are characterized by complex mechanisms involving interacting polyions, counterions and co-ions. Over the past decade there has been considerable interest in the static and dynamic properties of such systems from both theoretical and experimental points of view. Theoretical models include : crystal-solution of rods [1], notion of correlation hole [2-5], concept of electrostatic persistence length [6-8], counterion condensation [9], mode-mode coupling theory [10-12], entangled solution behavior $[13,14]$, phase transitions $[15,16]$ and weakly charged polyelectrolyte solutions based on the random phase approximation [17]. Various experimental techniques have been used to understand the behavior of such charged complex systems : small angle X-

$\left(^{*}\right)$ To whom correspondence should be addressed.

(**) On Sabbatical leave from Physics department, Tulane University, New Orleans, LA 70118 USA. 
rays [18, 19] and neutron [20, 21] scattering, elastic and quasi elastic light scattering [22, 33], and viscosity [34-37].

A question which seems to have plagued researchers involved with dilute polyelectrolyte solutions is what type of «structure» or « organization» exists in polyelectrolyte solutions without added salt or at low ionic strength. A variety of hypotheses, involving high degrees of long-range order, no long-range order, domain formation, exotic mechanisms for interaction, conventional mechanisms of interaction, « extraordinary regimes » of diffusion, absence of " extraordinary regimes », etc. have marked the work in this area during the past decade.

Rather than reviewing and comparing the various theories, the present work is offered as a case study in which it is unnecessary to involve notions of long-range order, « extraordinary regimes ", domain formation or any other unusual mechanism involved in the properties of these dilute polyelectrolyte solutions. These results are consistent with the body of evidence produced over the last several years [24-28, 38, 39].

Specifically, the broad angular scattering maxima found for dilute polyelectrolyte solutions in this study are most simply interpreted in terms of ordinary liquid-like correlations, in which, due to their electrostatically enhanced volume, there are strong repulsive interactions between nearest neighbors. Although not observed in this work, there might be very weak secondary and even feebler tertiary maxima, corresponding to weak correlations between second and third neighbors, just as in normal liquids. One notes, as we shall show later, that all these polyelectrolyte features can be observed only when all possible aggregates are removed by filtration on a proper type of membrane and pore size.

\section{Experimental section.}

2.1 MATERIALS, METHOdS ANd SAMPLE PREPARATION. - Succinoglycan (SG) is an exocellular polysaccharide produced by the bacteria Pseudomonas $s p$. NCIB 11592. Its repeat unit is composed of D-glucose:D-galactose:pyruvate:succinate in molar ratio of 7:1:1:1; in some cases the content of pyruvate and succinate can be modified by experimental conditions [40,41]. The chemical structure of the repeat unit is :

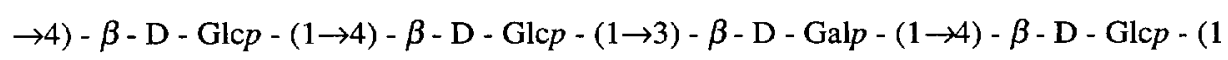

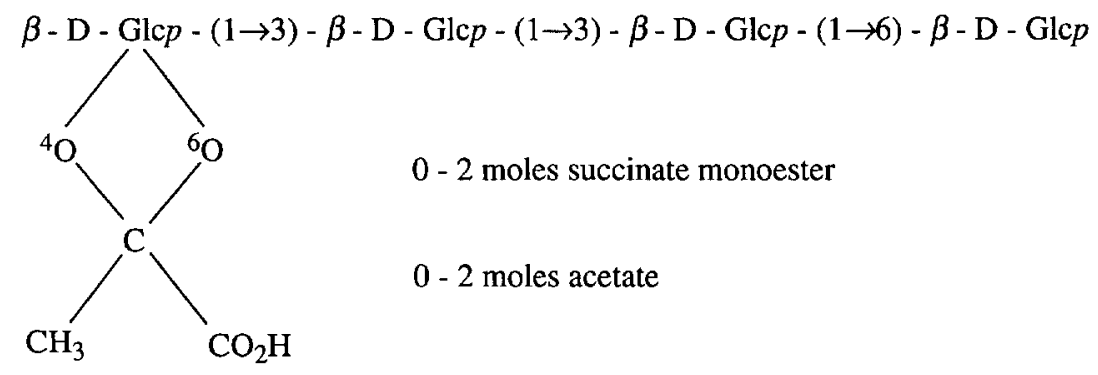

In dilute and salt-free solution this polysaccharide adopts a disordered conformation but in presence of external salt its conformation is a single helical chain [40].

The starting material was a bacterial broth supplied by Shell Research limited (Sittingbourne Research Center, Sittingbourne, Kent England). The samples were obtained by alcohol 
precipitation and subsequent reduced pressure room temperature drying, as has been already described [40, 41].

In this study, three different SG samples were studied. Virtually all the results presented here are from a fairly narrow molecular weight distribution of sonicated SG. Similar scattering behavior in all aspects was found also for the native SG sample. We concentrated on the sonicated sample because the lower mass solutions were easier to filter and handle.

Pure water was doubly distilled, de-ionized and filtered in a Millipore Alpha-Q; its conductivity was less than $0.05 \mu \mathrm{S}$ corresponding to an equivalent $\mathrm{NaCl}$ concentration of around $3.3 \times 10^{-7} \mathrm{M}$. SG solutions were allowed to dissolve and equilibrate few days prior to filtration and measurements.

The characteristics of SG in its disordered conformation corresponds to one charge every $16.8 \AA$, which gives a linear charge parameter $\lambda=0.42$ as deduced from the chemical structure in the Na-salt form. The molar mass per unit length is $66.7 \mathrm{~g} / \AA$.mol. These values were obtained from the ${ }^{1} \mathrm{H}$ NMR spectrum in $\mathrm{D}_{2} \mathrm{O}$ in the presence of an internal standard which gave 0.98 pyruvate and 0.24 succinate groups/repeat unit. In this disordered form the contribution to the total salt concentration due to the $\mathrm{SG}$ counterions and bare polyelectrolyte is roughly $C_{\mathrm{s}, \mathrm{c}}$ [equi/1] $=0.864 \times C_{\mathrm{p}}\left(\mathrm{g} / \mathrm{cm}^{3}\right)$. In excess of salt, the $\mathrm{SG}$ macromolecule adopts and extended helical conformation (stabilized by intramolecular $\mathrm{H}$-bonds); the above values correspond to one charge every $15.65 \AA, \lambda=0.45$ and $73.7 \mathrm{~g} / \AA$ mol for the molar mass per unit length. The helix-coil transition is induced by temperature increase or $\mathrm{NaOH}$ addition. Like Xanthan gum, the transition from disordered to helical state of SG is only $10 \%$ of change in axial ratio. This explains why the molar mass/unit length and the charge parameter are slightly different in both conformations.

We give upper and lower estimates of the overlap concentration $C *$ based on the wellknown formula $C^{*}=M_{\mathrm{w}} /\left(N_{\mathrm{A}} 4 \pi R^{3} / 3\right)$, where $R$ is a characteristic dimension of the molecule and $N_{\mathrm{A}}$ is Avogadro's number. We use the GPC results $M_{\mathrm{w}}=7.74 \times 10^{5} \mathrm{~g} / \mathrm{mol}$. The lower limit is obtained by assuming the SG to be fully extended occupying a spherical volume whose radius $R$ is half the rod length. With $R=5800 \AA$, this gives $C^{*}=1.57 \times 10^{-6} \mathrm{~g} / \mathrm{cm}^{3}$. The upper limit makes the assumption that $R=\left\langle S^{2}\right\rangle^{1 / 2}$ where $\left\langle S^{2}\right\rangle^{1 / 2}$ is about $1100 \AA$, the roughly constant value obtained at all $C_{\mathrm{s}}$, as discussed below. This gives $C^{*}=2.3 \times 10^{-4} \mathrm{~g} / \mathrm{cm}^{3}$.

To eliminate dust and other large particles, all samples were filtered. Both the pore size and material composition of the membrane filters used prior to light scattering experiments had large effects on the light scattering results. Due to the relative solvent affinity and the degree of swelling, the type of membrane (material) as well as the effective pore diameter indeed may play an important role on the filtration. All filters were $2.5 \mathrm{~cm}$ diameter membranes held in a stainless steel holder. The holders were attached via Luer lock fittings to glass syringes and steady manual pressure was used to filter the solutions. Three different types of filters were used : 1) all solutions not otherwise noted were filtered through $0.1 \mu \mathrm{m}$ cellulose nitrate filters (CN) (Sartorius, W-3400, Germany). 2) Comparisons with filtration through $0.2 \mu \mathrm{m}$ cellulose nitrate filters were also made (Sartorius), as well as comparisons with 3) $0.1 \mu \mathrm{m}$ polyvinylidene difluoride (PVDF) filters from Millipore which are more hydrophobic than the CN membranes. Concentration losses upon filtering were estimated by different techniques (UV absorption and conductivity) and found to be roughly on the order of $10 \%$. All concentations in this work are expressed in terms of weighed, dry material per $\mathrm{ml}$ of solvent, and thus represent an upper limit.

\subsection{EQUIPMENT AND DATA ANALYSIS.}

2.2.1 Static and dynamic light scattering. - The elastic and quasi-elastic scattering measurements were performed using the ALV (Langen-FRG) apparatus with an automatic goniometer 
table, a digital ratemeter and a temperature controlled sample cell. Temperature was $25 \pm 0.1^{\circ} \mathrm{C}$ unless otherwise noted.

The scattered light of a vertically polarized $\lambda_{0}=4880 \AA$ argon laser (SpectraPhysics $2020,3 \mathrm{~W}$, operating around $0.3 \mathrm{~W}$ ) was measured at different angles in the range of $20-150^{\circ}$ corresponding to $0.6 \times 10^{-3}<q / \AA<3.3 \times 10^{-3}$ where $q=\left(4 \pi n / \lambda_{0}\right) \sin (\theta / 2)$, $\theta$ the scattering angle, $n$ the refractive index of the medium $(n=1.33)$. The reduced elastic scattering $I(q) / k C_{\mathrm{p}}$, with $k=4 \pi^{2} n_{0}^{2}(\mathrm{~d} n / \mathrm{d} c)^{2}\left(I_{0}^{90^{\circ}} / R^{90^{\circ}}\right) / \lambda_{0}^{4} N_{\mathrm{A}}$, was measured in steps of $5^{\circ}$ in the scattering angle, where $n_{0}$ is the refractive index of standard (toluene), $I_{0}^{90^{\circ}}$ and $R^{90^{\circ}}$ are respectively the intensity and the Rayleigh ratio of the standard at $\theta=90^{\circ}$, $(\mathrm{d} n / \mathrm{d} c)$ the increment of refractive index, $C_{\mathrm{p}}$ the polyelectrolyte concentration, expressed in $\mathrm{g} / \mathrm{cm}^{3}$ and $I(q)$ the intensity scattered by the polymer. All elastic intensities were calculated according to standard procedures using toluene as reference with known absolute scattering intensity.

Because the total scattering intensity of the highly dilute SG in salt-free solution is scarcely above the scattering level of pure water the slightest amount of scattering impurities (e.g. «dust ») led to unacceptably noisy data. Consequently, an algorithm was written to extract the minimum intensity reading from a group of typically 50 separate intensity readings at each angle which are stored to disk by the ALV static data gathering program. The experimental and theoretical justification for this procedure has been previously given [24].

The full homodyne autocorrelation functions of the scattered intensity, also measured in steps of $5^{\circ}$ in the scattering angle, were obtained using the ALV-5000 autocorrelator from ALV, Langen, FRG. The intermediate scattering function $S(q, t)$ is related to the measured homodyne intensity-intensity time correlation function by the Siegert-relation [42]

$$
G^{(2)}(q, t)=B\left[1+\alpha|S(q, t)|^{2}\right]
$$

where $B$ is the base line and $\alpha$ is the spatial coherence factor depending upon the geometry of the detection and the ratio of the intensity scattered by the polymer to that scattered by the solvent. The autocorrelation functions of the scattered intensity were analyzed by means of the cumulant method to yield the effective diffusion coefficient as a function of the scattering angle. Additionally, inverse Laplace transform (ILT) and constrained regularization method (Contin) developed by Provencher [43] were used to obtain the distribution $A(\tau)$ of decay times. For the latter, a statistical parameter «probability to reject " is calculated for each solution, and the suggested one is that closest to 0.5 .

$$
\left[\frac{G^{(2)}(q, t)}{B}-1\right]^{1 / 2} \approx \int_{0}^{\infty} A(\tau) \mathrm{e}^{-(1 / \tau)} \mathrm{d} \tau
$$

These methods are now routinely used to analyze the quasi elastic light scattering (QELS) data for polymer systems and allows the determination of the relaxation modes which characterize the dynamic of such systems.

Static experiments were performed with a wide aperture for the phototube ( $300 \mu \mathrm{m}$ pinhole), whereas the dynamic experiments used the narrowest possible (typically $100 \mu \mathrm{m}$ in this range of concentration).

2.2.2 Size exclusion chromatography. - Size Exclusion Chromatography (SEC) characterization was carried out using a Waters 150C ALC/GPC with multiple detectors ; a single capillary viscometer [44], a Wyatt Technology Dawn F multi-angle laser light scattering detector (MALLS) with a vertically polarized He-Ne laser at $\lambda_{0}=6320 \AA$, and a refractive index detector built into the Waters unit. The simultaneous interfacting and operation of this system, as well as problems and procedures in data analysis will be presented in a 
forthcoming paper. With specially written software the Dawn F was also used for «batch " measurements on the SG; i) Zimm plots, ii) determination of $\left\langle S^{2}\right\rangle^{1 / 2}$ and second virial coefficients, $A_{2}$, versus salt concentration $C_{s}$, and iii) dimensions and $A_{2}$ versus $[\mathrm{NaOH}]$.

2.2.3 Viscosity measurements. - The reduced viscosity $\eta_{\mathrm{r}}=\left(\boldsymbol{\eta}-\eta_{0}\right) / \eta_{0} C_{\mathrm{p}}$, where $\eta_{0}$ is the solvent viscosity, measurements were made with a Low Shear 30 cylinder in cup viscometer. This allowed measurements to be made on the Newtonian plateau. The viscosity measurements made in pure water as solvent in the range of polymer concentration from $10^{-5} \mathrm{~g} / \mathrm{cm}^{3}$ to $10^{-3} \mathrm{~g} / \mathrm{cm}^{3}$, which includes the domain over which the light scattering experiments were performed. These solutions were also filtered through $0.1 \mu \mathrm{m}$ and $0.2 \mu \mathrm{m}$ cellulose nitrate membranes.

\section{Results and discussion.}

3.1 SEC RESULTS. - Figure 1 shows SEC results for filtered through $0.1 \mu \mathrm{m}$ cellulose nitrate. using the increment of refractive index $(\mathrm{d} n / \mathrm{d} c)=0.154$ for SG in $100 \mathrm{mM} \mathrm{NH}_{3} \mathrm{NH}_{4}$ yielded number, weight and $z$-average molecular weights $M_{\mathrm{n}}, M_{\mathrm{w}}, M_{\mathrm{z}}$ respectively given in the figure. The slope of the root mean square radius of gyration $\left\langle S^{2}\right\rangle^{1 / 2}$ is $0.584+1-0.04$. The intrinsic viscosity was around $2600 \mathrm{~cm}^{3} / \mathrm{g}$.

Using the coil limit of the worm-like chain formula $\left\langle S^{2}\right\rangle=L L_{\mathrm{T}}^{\prime} / 3$ where $L$ is the contour length of the molecule, allows apparent persistence length $L_{\mathrm{T}}^{\prime}$ to be estimated from the data in figure 1. One notes that this so-called apparent persistence length includes the electrostatic contribution as well as the excluded volume effect. It has been defined and discussed elsewhere [24-27, 45-47]. This value is around $300 \AA$ at $C_{\mathrm{s}}=100 \mathrm{mM}$. This is in good agreement with earlier report [48] and shows that $S G$ is a rather stiff polymer, in the class of xanthan, for example, and quite a bit stiffer than hyaluronate $\left(L_{0}=87 \AA\right.$, where $L_{0}$ is the intrinsic persistence length, estimated by extrapolation of $L_{\mathrm{T}}^{\prime}$ to infinite ionic strength). With $L_{\mathrm{T}}^{\prime}=300 \AA$ for SG, the number of Kuhn segments is about 19. This value is not far from that of the coil limit for static dimensions, and certainly too many for the SG to be considered as a rod at this salt concentration.

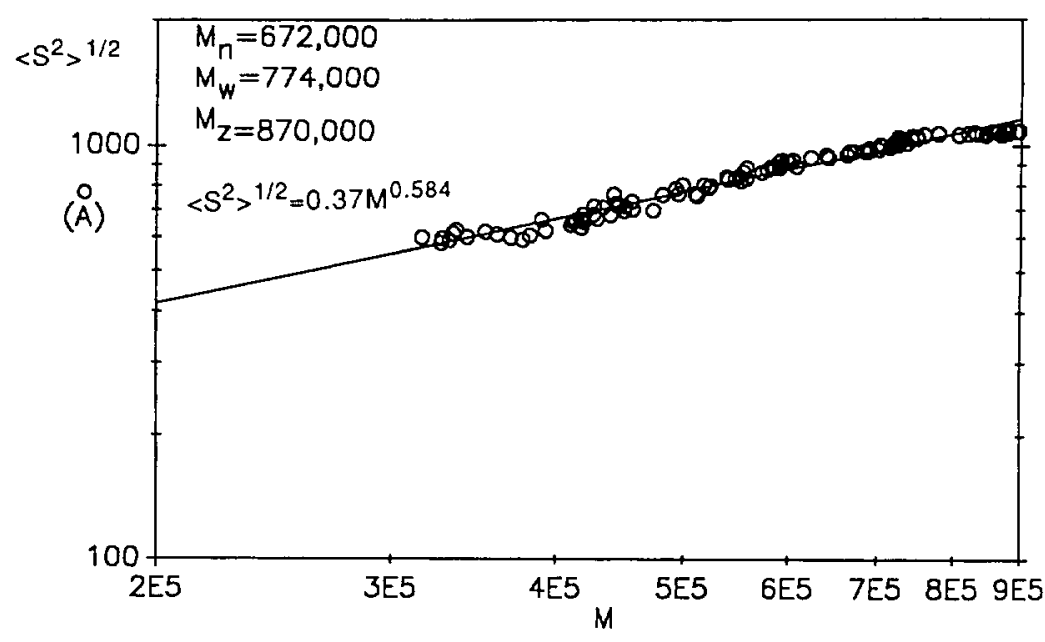

Fig. 1. - SEC results for SG at $C_{\mathrm{s}}=0.1 \mathrm{M} . M_{\mathrm{n}}, M_{\mathrm{w}}$ and $M_{\mathrm{z}}$ are given. 
3.2 Dimensions AND $A_{2}$ versus. $C_{3}$. - Figure 2 a shows the slope of $k C_{\mathrm{p}} / I(q)$ versus $q^{2}$ for SG at $0.1 \mathrm{mg} / \mathrm{ml}$ for different values of $C_{\mathrm{s}}$ (from serial additions of small amounts of $\mathrm{NaCl}$ stocks). For reasons explained below, the slope is initially negative, and becomes flatter, and finally positive as the salt concentration $C$, increases. By about $1 \mathrm{mM}$ the slope is nearly zero and then becomes positive and remains constant thereafter. It is only after the slope has become positive, and electrostatic interactions between polymers sufficiently suppressed, at about $4 \mathrm{mM}$ that one can use standard static light scatering techniques to estimate $\left\langle S^{2}\right\rangle^{1 / 2}$ and $A_{2}$.

Interestingly, the dimension of the SG in the accessible $C$, range (after about $4 \mathrm{mM}$ ) is quite insensitive to $C_{\mathrm{s}}$. The estimated values of $\left\langle S^{2}\right\rangle$, calculated from the slopes (at $\left.C_{\mathrm{s}}\right\rangle 4 \mathrm{mM}$ ), are shown in figure $2 \mathrm{~b}$. The solid line shows the calculation of $\left\langle S^{2}\right\rangle$ according to a combination of electrostatic persistence length (EPL) and electrostatic excluded volume (EEV) theories, which has given fairly good predictions of $\left\langle S^{2}\right\rangle$ and $A_{2}$ versus $C_{\mathrm{s}}^{-1 / 2}$ and $C_{s}$, respectively, with no adjustable parameters [24-27, 46, 47].

Although the calculated curve shows only a modest decrease of $\left\langle S^{2}\right\rangle^{1 / 2}$ with increasing $C_{s}$, the experimental data show no change. The reason for this intensitivity of $\left\langle S^{2}\right\rangle^{1 / 2}$ to $C_{\mathrm{s}}$ is unclear. Aside from experimental uncertainty, it may be due, for example, to residual electrostatic effects for the low $C_{\mathrm{s}}$ values which tend to depress the slope against the effect of coil expansion (Fig. 2a) which, contrarily, increases the slope.

Another possibility is that at the lower ionic strength the SG is in a mixture of helicoidal and disorganized states. The apparent intrinsic persistence length of $\mathrm{SG}$ in the disorganized forms is only about $50 \AA$ [48]. According to EPL/EEV computations, SG dimensions in the disordered state at these ionic strengths would be smaller than those of the helical SG, the average radius of gyration being measured thus being less than that of the purely helical form.

In contrast, figure $2 \mathrm{c}$ shows how sensitive $A_{2}$ is to added salt concentration $C_{\mathrm{s}}$. indicating appreciable intermolecular electrostatic excluded volume effects. The solid line in figure $2 c$ shows the calculation of $A_{2}$ via the same combined EPL and EEV theories. It is emphasized that this calculation, which predicts $A_{2}$ quite well, involves no adjustable parameters. It is also noted that virtually the whole variation of $\left\langle S^{2}\right\rangle$ over this range is due to EEV effects, the intrinsic stiffness of SG making the EPL contribution negligible over this range. Thus, it seems that while the interactions between polymer chains which are controlled by the electrostatic field are highly sensitive to $C$, from zero added salt up to $0.1 \mathrm{M}$, as they should be, the dimensions are insensitive.

3.3 CONTRACTION OF SG WITH SODIUM HYDROXYDE (NAOH). - In a preliminary effort to understand the high inherent rigidity of $\mathrm{SG}$, the behavior of $\left\langle S^{2}\right\rangle^{1 / 2}$ versus $[\mathrm{NaOH}]$ was measured. The solution was originally at $C_{\mathrm{s}}=0.1 \mathrm{M} \mathrm{NaCl}$ before serial dilution with $\mathrm{NaOH}$. This is given in figure 3, where it is seen that the $S G$ undergoes a contraction of size as [NaOH] increases. The weight-average apparent persistence length $L_{\mathrm{T}}^{\prime}$ decreases from about 318 to $100 \AA$ as $\mathrm{NaOH}$ increases. This suggests that hydrogen bonds, which might stabilize helical segments of $\mathrm{SG}$ at lower $\mathrm{pH}$, are being broken as the $-\mathrm{OH}$ groups on the saccharide rings are deprotonated to $-\mathrm{O}^{-}$at high $\mathrm{pH}$, leading to a loss of rigidity. The shape of the curve in figure 3 is remarkably similar to a titration curve, further strengthening the notion that it is the $\mathrm{pH}$ controlled deprotonation of $-\mathrm{OH}$ groups which control the inherent SG stiffness. All the same time (for $[\mathrm{NaOH}]>0.1 \mathrm{M}$ or $\mathrm{pH}>13$ ) the deprotonation will progressively increase the charge parameter without any effective role on the electrostatic expansion which is due to the high ionic concentration of the solution. Further evidence of this contraction comes from the behavior of $A_{2}$ versus $\mathrm{NaOH}$, also shown in figure 3. Since $A_{2}$ essentially measures polymer volume per unit mass at high $C_{\mathrm{s}}, A_{2}$ should decrease concommittantly with $\left\langle S^{2}\right\rangle^{1 / 2}$, as is the 

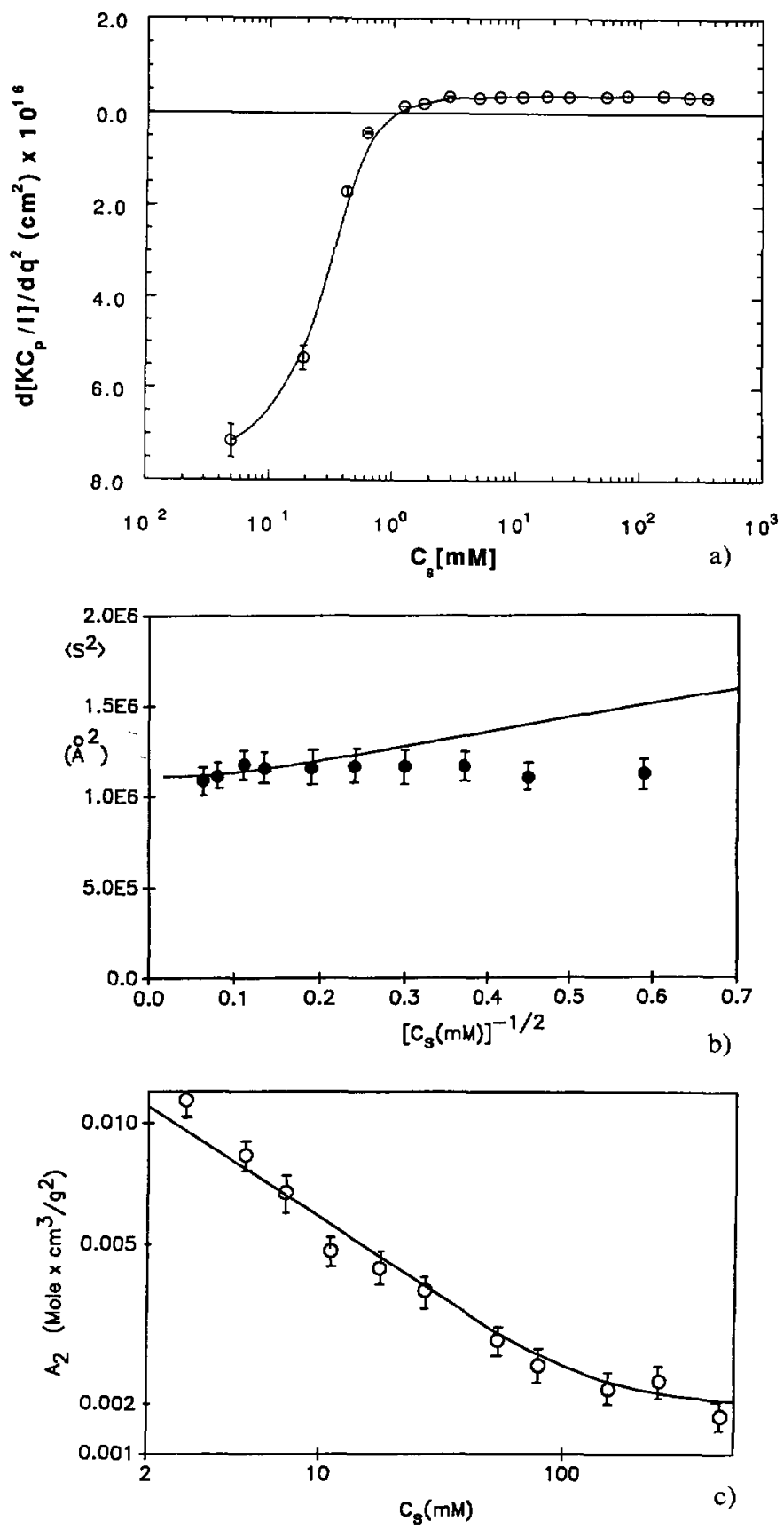

Fig. 2. - a) Slope of $k C_{\mathrm{p}} / I(q)$ versus $q^{2}$ for different $C$, for $C_{\mathrm{p}}=0.1 \mathrm{mg} / \mathrm{ml}$. The negative slope at very low $C$, is due to the range of $q$ measured being on the increasing side of the $I v s . q$ peak. The peak itself is at too high a $q$ value to be measured with visible light. b) $\left\langle S^{2}\right\rangle$ versus $(C,\rangle^{-1 / 2}$, calculated from the positive slopes in figure $2 \mathrm{a}$. There is almost no dependence of the radius of gyration on $C$, once the slope of $k C_{\mathrm{p}} / I(q)$ becomes positive. The solid line shows the calculation of $\left\langle S^{2}\right\rangle$ versus $C_{5}^{-1 / 2}$ according to combined EPL and EEV theories [24-27.46]. c) Variation of $A_{2}$ as function of $C_{\text {, }}$ as obtained from the intercepts of $k C_{\mathrm{p}} / I$ versus $q^{2}$. The solid line is the result of combined EPL and EEV theories with no adjustable parameters [24-27, 46]. 


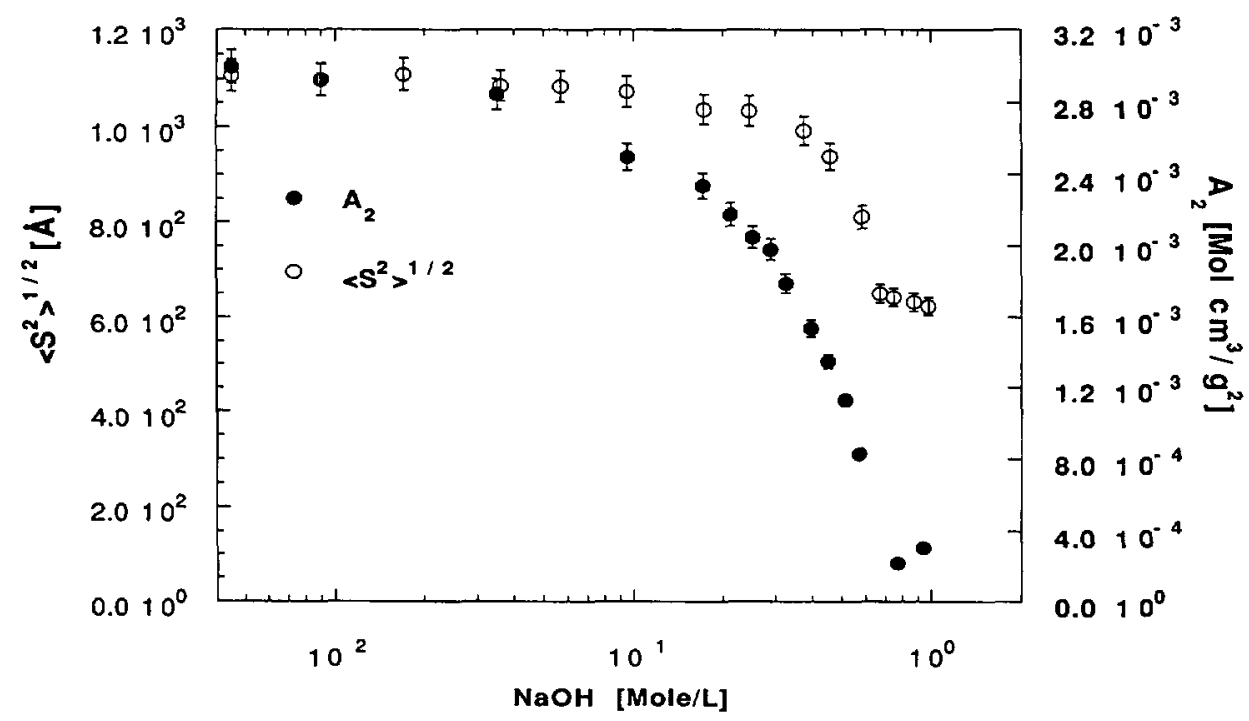

Fig. 3. - Variation of $\left\langle S^{2}\right\rangle^{1 / 2}$ and $A_{2}$ versus $[\mathrm{NaOH}]$. The solution was originally at $C_{\mathrm{s}}=0.1 \mathrm{M} \mathrm{NaCl}$. It shows large contraction of the molecule with $\mathrm{NaOH}$.

case in figure 3. These results are consistent with recent results for hyaluronate [49], which showed a similar loss of stiffness versus $[\mathrm{NaOH}]$ in a titration curve fashion. From $\left\langle S^{2}\right\rangle^{1 / 2}$ and $A_{2}$ in figure 3 , the pKa of the $-\mathrm{OH}$ group can be estimated to be roughly 13.7. The contraction of $\mathrm{SG}$ is not due to degradation. Solutions of $\mathrm{SG}$ at $1 \mathrm{M} \mathrm{NaOH}$ showed no degradation after $15 \mathrm{~h}$.

\subsection{STATIC AND DYNAMIC LIGHT SCATTERING.}

3.4.1 Salt-free solutions. - In the absence of added salt, solutions of SG at extremely low concentrations, filtered through $0.1 \mu \mathrm{m}$ cellulose nitrate $(\mathrm{CN})$ filters manifested clear static angular scattering peaks. Broad but well defined peaks appear at certain $q_{\mathrm{m}}$ whose values depend on the polyelectrolyte concentration. Figure 4 shows typical examples of these peaks for several concentrations ranging from $0.65 \times 10^{-5} \mathrm{~g} / \mathrm{cm}^{3}$ to $4.53 \times 10^{-5} \mathrm{~g} / \mathrm{cm}^{3}$ where $I / k C_{\mathrm{p}}$ is plotted against $q$.

There is perhaps a hint of a second maximum, not very well defined, at the lowest investigated concentration $C_{p}=0.65 \times 10^{-5} \mathrm{~g} / \mathrm{cm}^{3}$. Since this possible low peak falls within experimental error we do not pursus any further analysis.

In this range of concentration, the scattering vectors of the peak maxima scales as $C_{\mathrm{p}}^{0456 \pm 002}$ as displayed in figure 5 (curve 1). This exponent is consistent with the notion of correlation hole effect [2-5], and liquid-like correlations of cylindrical scatterers [27, 38]. Although the SG does not appear to be in the rod limit under these conditions it is perhaps locally stiff enough, and on the whole anisotropic enough that inter-molecular interactions resemble rod-rod encounters in the region of contact. Our above estimates lead us to believe that the range of concentrations for which peaks were observed may still be close to the semidilute regime, despite the extremely low concentrations. The fact that fits of $q_{\mathrm{m}}$ versus $C_{\mathrm{p}}$ always gave exponents slightly less than 0.5 may indicate, however, we are near the dilute to semi-dilute transition regime. A clear elbow of $q_{\mathrm{m}}$ versus $C_{\mathrm{p}}$ with a slope passing from $1 / 2$ 


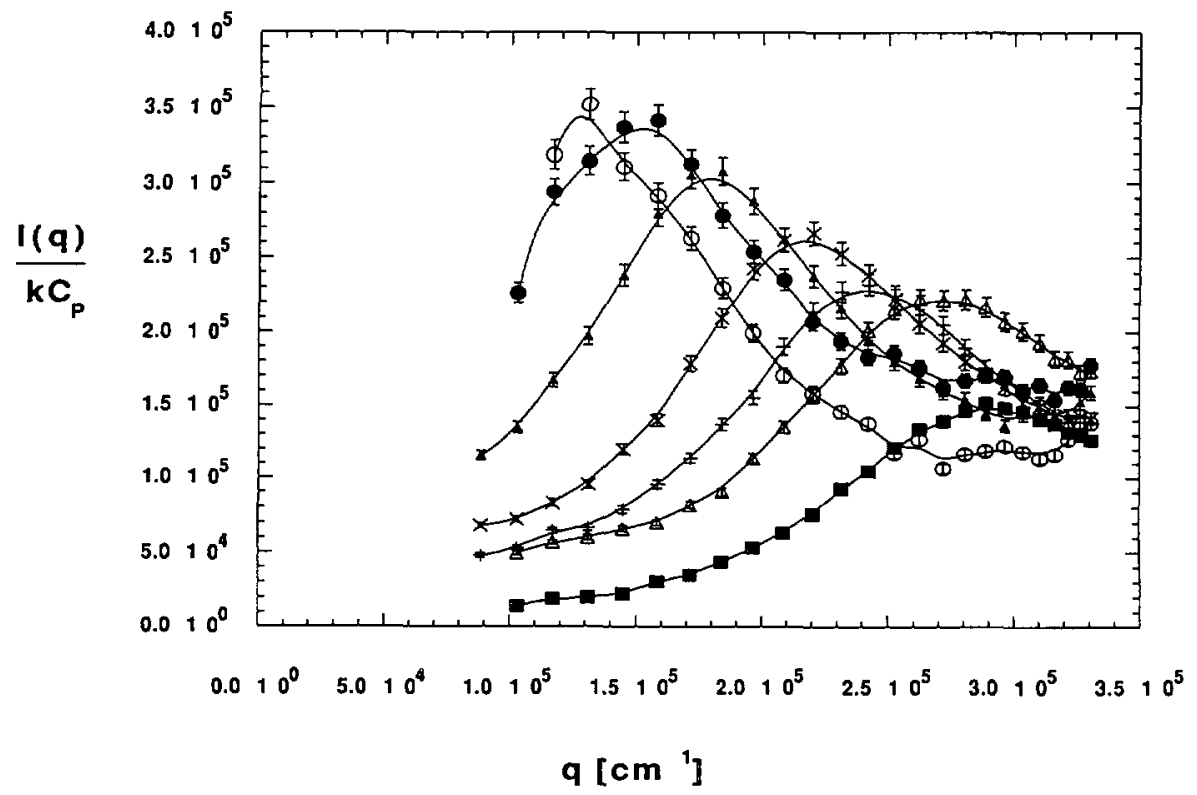

Fig. 4. $-I(q) / k C_{p}$ versus $q$ for salt-free solutions of SG at various polyelectrolyte concentrations $C_{\mathrm{p}}$.(O) $0.65 \times 10^{-5} \mathrm{~g} / \mathrm{cm}^{3} ;(\bullet) 1.01 \times 10^{-5} \mathrm{~g} / \mathrm{cm}^{3} ;$ (४) $1.59 \times 10^{-5} \mathrm{~g} / \mathrm{cm}^{3} ;(\times) 2.38 \times 10^{-5} \mathrm{~g} / \mathrm{cm}^{3}$ : $(+) 3.03 \times 10^{-5} \mathrm{~g} / \mathrm{cm}^{3} ;(\triangle) 3.84 \times 10^{-5} \mathrm{~g} / \mathrm{cm}^{3} ;(\square) 4.53 \times 10^{-5} \mathrm{~g} / \mathrm{cm}^{3}$.

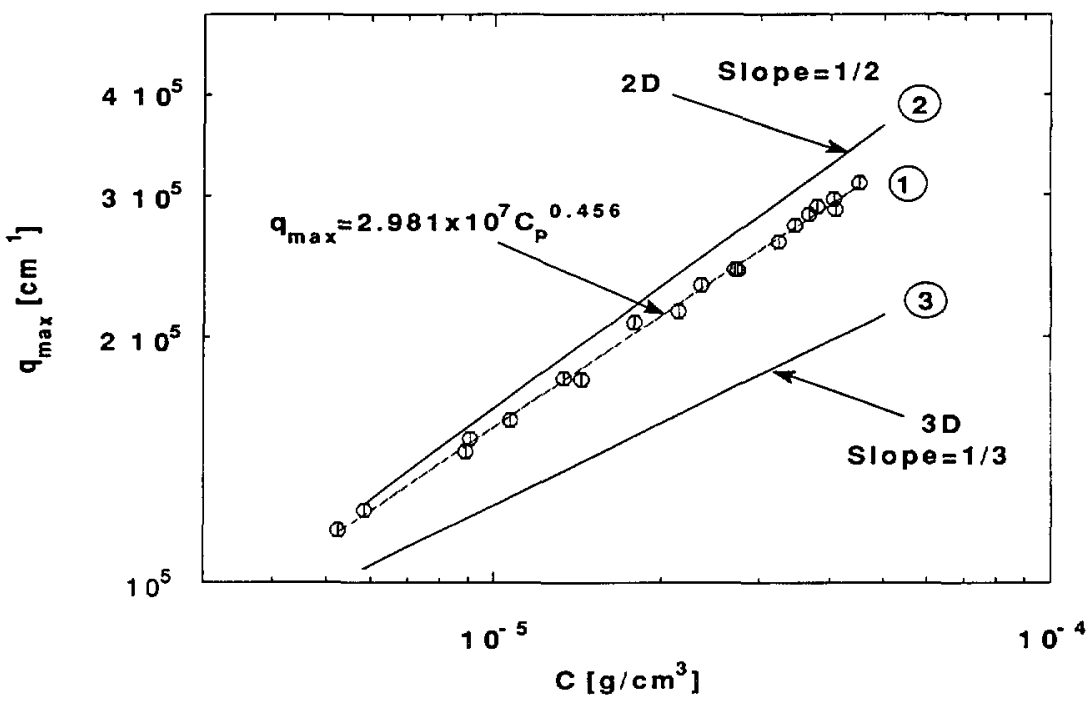

Fig. 5. - Variation of $\log \left[q_{\mathrm{m}}\right]$ as a function of $\log \left[C_{\mathrm{p}}\right]$ (curve 1). A $10 \%$ concentration loss due to filtration is assumed. Curve 2 corresponds to rod limit, hexagonal 2D packing $q_{\mathrm{m}}=2 \pi\left[3(\mathrm{~d} / \mathrm{m}) C_{\mathrm{p}}\left(\mathrm{g} / \mathrm{cm}^{3}\right) N_{\mathrm{A}} / 4\right]^{1 / 2}$, and curve 3 corresponds to a cubic arrangement of scatterers in the dilute regime, $q_{\mathrm{m}}=2 \pi\left[C_{\mathrm{p}}\left(\mathrm{g} / \mathrm{cm}^{3}\right) N_{\mathrm{A}} / M\right]^{1 / 3}$ 
in the semi-dilute regime to $1 / 3$ in the dilute regime has been demonstrated in several works for hard cylinders [38], DNA [50] and the semi-flexible polyelectrolyte sodium polystyrene sulfonate (NAPSS) [39].

Assuming a hexagonal 2D arrangement of infinitely long cylinders (although the SG is not expected to be in the rod limit) leads to a prediction of $q_{\mathrm{m}}=2 \pi\left[3(\mathrm{~d} / \mathrm{m}) C_{\mathrm{p}}\left(\mathrm{g} / \mathrm{cm}^{3}\right) N_{\mathrm{A}} / 4\right]^{1 / 2}$ where $(d / \mathrm{m})$ is the distance/molar mass $(\mathrm{cm} . \mathrm{mol} / \mathrm{g})$ of the polymer, taken as $\left(1.45 \times 10^{-10} \mathrm{~cm} . \mathrm{mol} / \mathrm{g}\right)$ in this case. This corresponds to curve (2) in figure 5 . It is stressed that this simple picture is a means of estimating average distances between idealized rods in the semi-dilute regime and in no way is meant to imply that liquid crystals or other types of static, organized domains exist. Assuming a cubic arrangement of scatterers in the dilute regime gives $q_{\mathrm{m}}=2 \pi\left[C_{\mathrm{p}}\left(\mathrm{g} / \mathrm{cm}^{3}\right) N_{\mathrm{A}} / M\right]^{1 / 3}$, where $M$ is the molecular weight of the polyelectrolyte. This function for the SG with $M=7.74 \times 10^{5}$ is also shown in figure 5. Taking account of possible concentration losses on the order of ten percent yields curve (3) in figure 5 for the cylindrical packing estimate. In this respect, the effect of the molecular weight on the slope is in progress in our laboratory.

At this level one notes that the negative values of the slopes reported in figure $2 \mathrm{a}$ are due to $C_{\mathrm{p}}$ being high enough and the $I(q)$ peak occurs above the highest accessible $C_{\mathrm{p}}\left(q_{\mathrm{m}}=\right.$ $4.47 \times 10^{5} \mathrm{~cm}^{-1}$ ) and the monotonically increasing $I(q)$ gives a negative slope for $k C_{\mathrm{p}} / I(q)$.

The shape and values of $I(q) / k C_{\mathrm{p}}$ versus $q$ were insensitive to time and only slightly sensitive to the temperature (over the range $12{ }^{\circ} \mathrm{C}$ to $50^{\circ} \mathrm{C}$ ). This behavior is illustrated in figure 6 . It seems that there is a slight broadening and a decrease in peak height with increasing temperature. This is consistent with a slight increase of thermal motion between scatterers. One notes that earlier reports have indicated that scattering peaks associated with latex spheres evolved and sharpened over a time scale of many hours or even days to crystallize into lattices with long range order [51]. The dashed curve in figure 6 shows the scattering peak remained

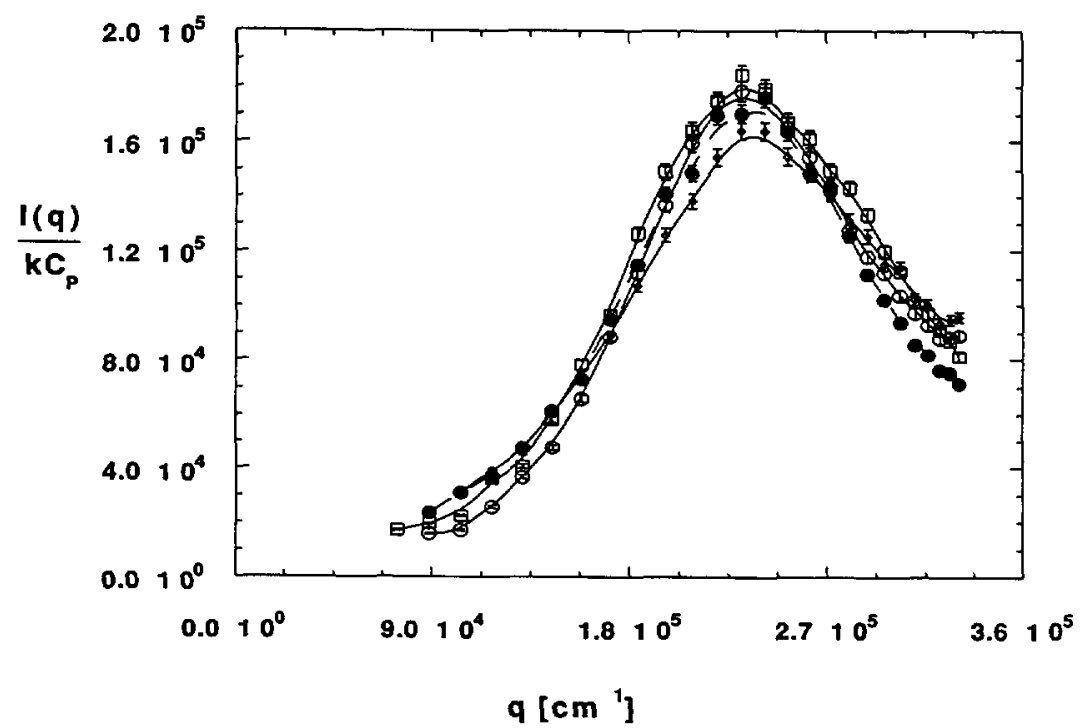

Fig. 6. - Variation of $I(q) / h C_{\mathrm{p}}$ as a function of $q$ for $C_{\mathrm{p}}=3 \times 10^{-5} \mathrm{~g} / \mathrm{cm}^{3} \mathrm{SG}$ with no added salt. The symbols $(\square, \varnothing, \bigcirc)$ show $I / k C_{\mathrm{p}}$ at $t=50{ }^{\circ} \mathrm{C}, t=25^{\circ} \mathrm{C}, \mathrm{t}=12{ }^{\circ} \mathrm{C}$, respectively and $(\bullet)$ shows the same solution after being left for 3 days at room temperature. No peak narrowing or other changes in the scattering suggest the liquid-like correlated state is an equilibrium state. 
unchanged after 4 days of remaining undisturbed at $t=25^{\circ} \mathrm{C}$. Thus, there is no evidence that the SG solutions "crystallize» into systems with long range order over the time and temperature scales studied here.

QELS experiments were also performed on salt-free SG solutions. The autocorrelation functions for solutions filtered through $0.1 \mu \mathrm{m}(\mathrm{CN})$ filters were described by a single exponential decay (see the discussion in the section below, «the problem of aggregates »).

Figure 7 shows the angular variation of the reciprocal diffusion coefficient $1 / D(q)$ deduced from the standard second order cumulant analysis of the autocorrelation functions.

$$
\ln |S(q, t)|=-\langle\Gamma\rangle \tau+\frac{\mu_{2}}{2 !} \tau^{2}-\frac{\mu_{3}}{3 !} \tau^{3}+\cdot
$$

where $\mu_{1}$ are the moments about the mean of the distribution $A(z)$.

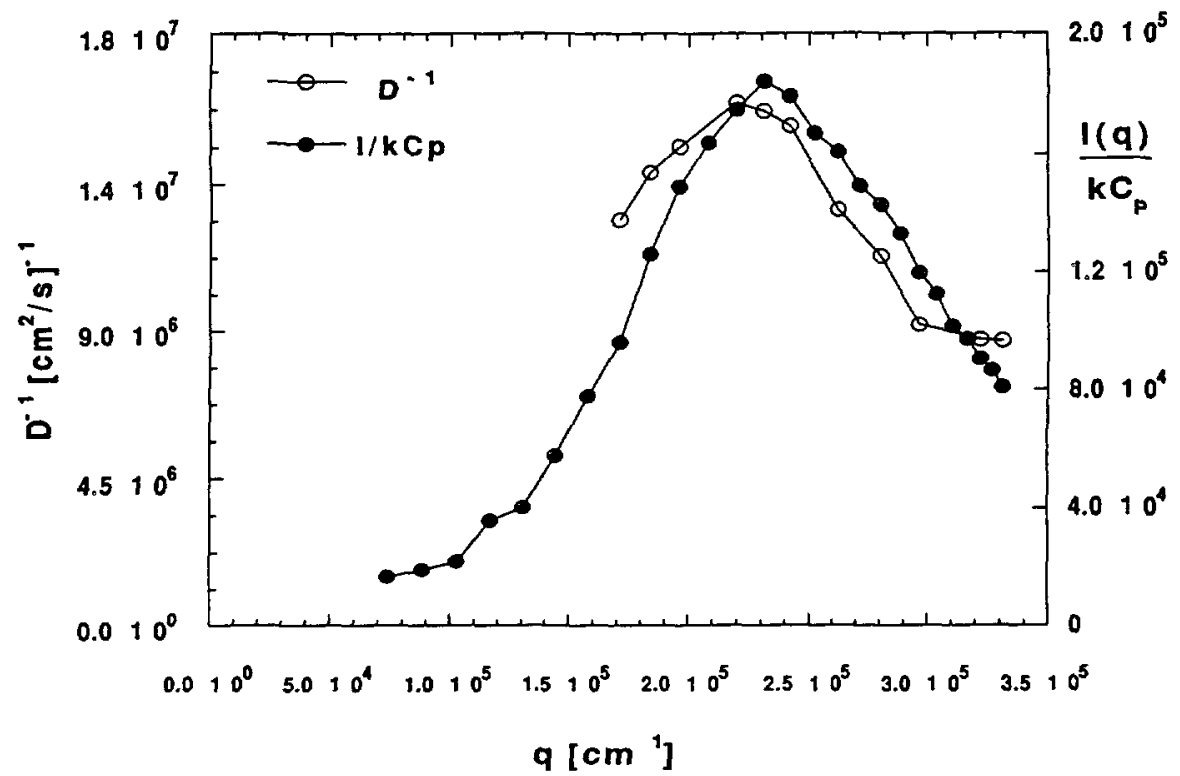

Fig. 7. - Angular variation of the reciprocal diffusion coefficient $D^{-1}$ computed from the standard second order cumulant analysis of the autocorrelation functions and $I / k C_{\mathrm{p}}$ at $C_{\mathrm{p}}=3.01 \times 10^{-5} \mathrm{~g} / \mathrm{cm}^{3}$, filtered through $0.1 \mu \mathrm{m} \mathrm{CN}$.

The solution was salt-free $\mathrm{SG}$ at $C_{\mathrm{p}}=3.01 \times 10^{-5} \mathrm{~g} / \mathrm{cm}^{3}$, filtered through $0.1 \mu \mathrm{m} \mathrm{CN}$. Below $\theta=50^{\circ}$, the autocorrelation functions were too noisy due to the weak scattering signal and occasional dust particles which scatter more strongly at low angles. On the same figure is also reported $I(q) / k C_{\mathrm{p}}$ for the same concentration. Both $I(q)$ and $1 / D(q)$ have qualitatively the same shape and approximately the maximum at the same $q$-position. This result shows that the mobility $M(q)$ is independent of $q$ according to the general Ferrel-Kawasaki expression for the apparent diffusion coefficient $D \approx M(q) / S(q)$, as has often been observed [22, 27, 52]. Depending on the range of concentration (dilute or semi-dilute) the difference between the detailed shapes of the peaks has been attributed to hydrodynamic effects [53].

3.4.2 Effect of added salt. - Addition of a simple electrolyte $(\mathrm{NaCl})$ to the solutions screens the electrostatic interactions and reduces the osmotic pressure. Figure 8 indicates the behavior

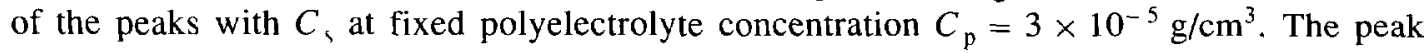
height decreases with $C_{s}$, and its position seems to remain constant. One observes that the 


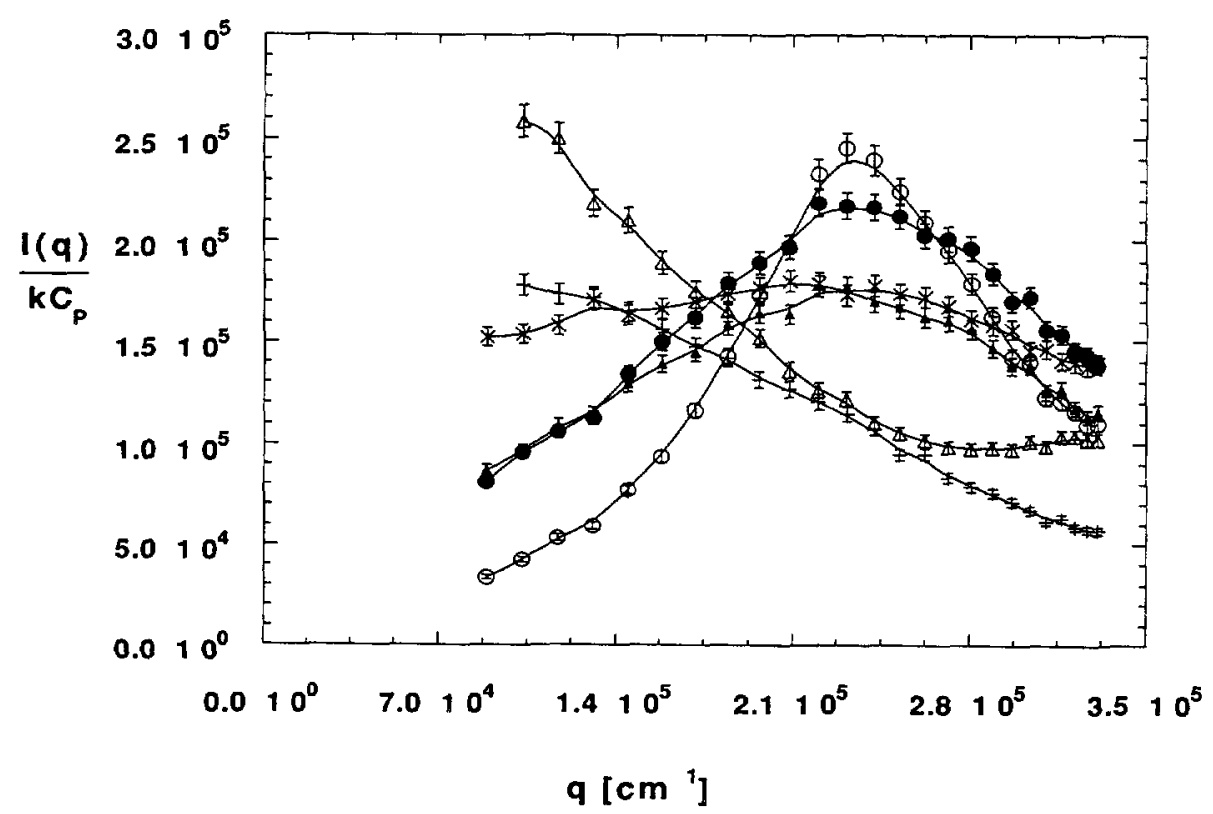

Fig. 8. - Variation of $I(q) / k C_{\mathrm{p}}$ as a function of $q$ for $C_{\mathrm{p}}=3.0 \times 10^{-5} \mathrm{~g} / \mathrm{cm}^{3} \mathrm{SG}$ at different added salt

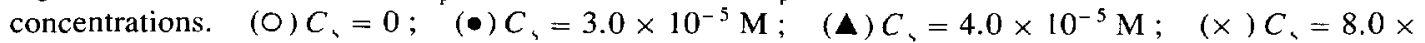
$10^{-5} \mathrm{M} ;(\Delta) C_{\mathrm{s}}=12 \times 10^{-5} \mathrm{M} ;(+) C_{\mathrm{s}}=40 \times 10^{-5} \mathrm{M}$.

region $q<q_{\mathrm{m}}$ is very sensitive to the addition of salt and that of $q>q_{\mathrm{m}}$ preserves a level of scattering close to the value in the absence of salt as expected by most theoretical models for electrostatic interaction in the semi-dilute range of concentration. However, one notes that as $C_{\mathrm{s}}$ increases this behavior is progressively lost and the reason is that the addition of salt shifts the system from semi-dilute to dilute according to the change of the molecule conformation.

When enough salt is added the scattering behavior is closer to that of a neutral polymer. For example this neutral type behavior is reached for $C_{\mathrm{p}}=3.0 \times 10^{-5} \mathrm{~g} / \mathrm{cm}^{3}$ at $C_{\mathrm{s}}=4 \times 10^{-4} \mathrm{~mol} / \mathrm{l}$ (see Fig. 8) and corresponds to the slopes in figure $2 \mathrm{a}$ above $C_{\mathrm{s}}=4 \mathrm{mM}$ for $C_{\mathrm{p}}=10^{-4} \mathrm{~g} / \mathrm{cm}^{3}$. The same behavior has been observed for proteoglycan monomer [27].

The effect of added salt concentration $C_{\mathrm{s}}$ on the dynamic behavior of the SG sample at $C_{\mathrm{p}}=3 \times 10^{-5} \mathrm{~g} / \mathrm{cm}^{3}$ is represented in figure 9 . The equivalent apparent hydrodynamic radius computed by the Stockes-Einstein equation from the second cumulant analysis is plotted as a function of $C_{3}$, where $C_{3}$ is the sum of roughly $0.026 \mathrm{mM}$ contribution of the $\mathrm{SG}$ counterion and the added salt $\mathrm{NaCl}$. No added salt corresponds to $C_{\mathrm{s}}=0.026 \mathrm{mM}$ in the figure. Two angles were chosen; $\theta=90^{\circ}$, which is the scattering peak, and $150^{\circ}$ which is far from the peak. For convenience the $D^{-1}$ value at the peak is converted to an hypothetical value of $R_{\mathrm{h}}$ and is seen to fall from $450 \AA$ at no added salt, to about $350 \AA$ at $0.014 \mathrm{mM}$ of added salt, and then to quickly fall to a constant value of around $200 \AA$. which converges with the value at $\theta=150^{\circ}$. The value at $\theta=150^{\circ}$ is fairly constant falling from about $235 \AA$ at no added salt to about $200 \AA$ at high $C_{\mathrm{s}}$. Since $D^{-1}(q)$ basically follows $I(q)$, the quick fall of $R_{\mathrm{h}}$ with $C_{\mathrm{s}}$ is consistent with the loss of the static peak as $C_{\mathrm{s}}$ increases in figure 8 .

$R_{\mathrm{h}}$ far away from the peak may be close to a true measure of the equivalent hydrodynamic radius, which scarcely varies with $C_{5}$. The magnitudes of $R_{\mathrm{h}}$ are not very close to what would be expected for a wormlike chain close to the coil limit : with an $R_{\mathrm{g}}=\left\langle S^{2}\right\rangle^{1 / 2}$ of about $1100 \AA$ 


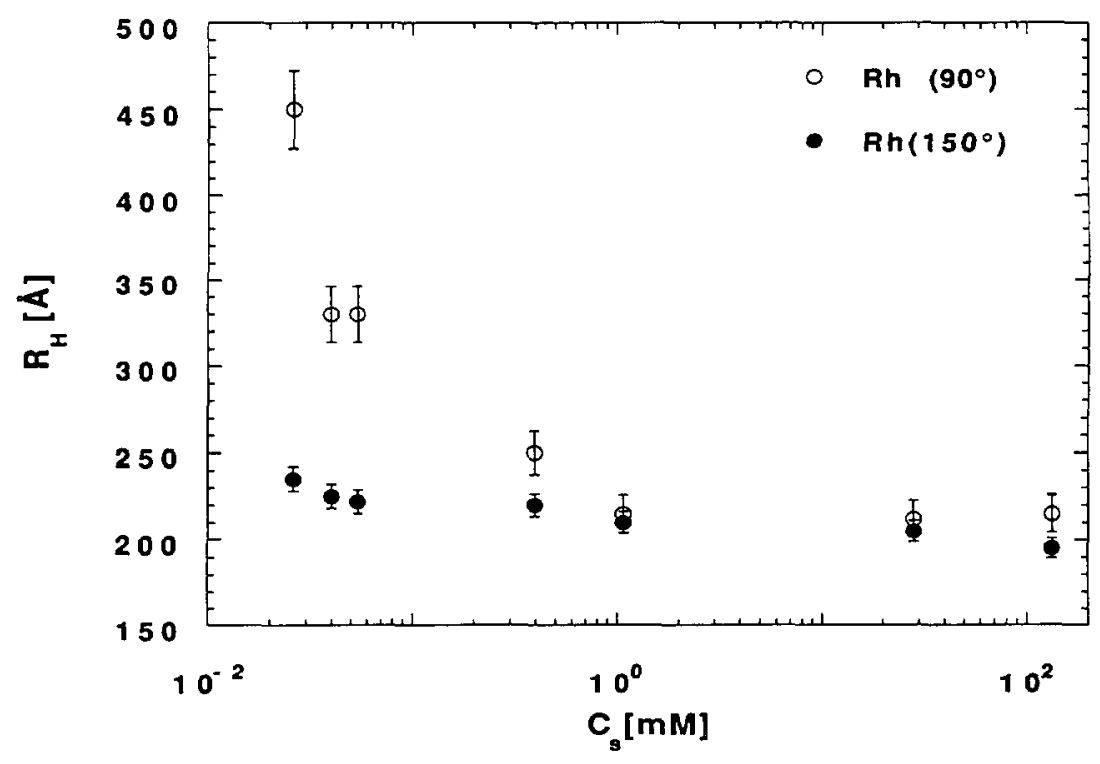

Fig. 9. - Variation of $R_{\mathrm{h}}$ as a function of $C_{\mathrm{s}}$ for $C_{\mathrm{p}}=3 \times 10^{-5} \mathrm{~g} / \mathrm{cm}^{3} \mathrm{SG}$ at two scattering angles $90^{\circ}$ (the peak of $R_{\mathrm{h}}$ ) and $150^{\circ}$.

an ideal coil would have an $R_{\mathrm{h}}$ of about $730 \AA$. The fact that $R_{\mathrm{h}}=210 \AA$ is lower than this value is consistent with similar hydrodynamic behavior for many linear polyelectrolytes [2426], where the ratio of $R_{\mathrm{h}} / R_{\mathrm{g}}$ is often observed to be much smaller than the ratio of 0.67 predicted for the non-draining coil limit. In this case $\left(R_{\mathrm{h}} / R_{\mathrm{g}}\right)=0.2$ which is close to the value for another semi-flexible polymer: xanthan $\left(\left(R_{\mathrm{h}} / R_{\mathrm{g}}\right)=0.35\right)$ [54]. This difference is suspected to be due to the rigidity of the chain which is probably at the origin of partial draining effect. In the case of the $\mathrm{SG}$ it is seen that $R_{\mathrm{h}} / R_{\mathrm{g}}$ remains constant for all measured values of $C_{\mathrm{s}}$. It should be pointed out that in the case of other linear polyelectrolytes such as hyaluronic acid [24], and variably ionized polyacrylate (polyacrylic acid) [25] there were large, measurable changes in $R_{\mathrm{g}}$ with $C_{\mathrm{s}}$, but none in $R_{\mathrm{h}}$, again perhaps attributable to some type of draining effect.

At any rate it is clear that the $R_{\mathrm{h}}=450 \AA$ at the peak and at zero added salt does not correspond to any structure of unusual dimensions, such as temporal aggregates, domains, etc. [55]. In fact, the magnitude and behavior of $R_{\mathrm{h}}$ with $C_{\mathrm{s}}$ does not resemble the « slow mode » of diffusion often reported for polyelectrolytes at low $C_{\mathrm{s}}[29,31-33,55]$. Only incomplete elimination of aggregates led to a « slow mode » is SG solutions, as is now discussed.

3.5 THE PROBLEM OF AGGREGATES. - The succinoglycan contained a relatively large amount of aggregates, probably already existing or nascent in the dry material, perhaps due to the method in which it was prepared. When filtering SG solutions through $0.2 \mu \mathrm{m}$ cellulose nitrate membranes the aggregate population dominated the scattering; there was high background scattering from the solution and the peak was far less pronounced. Only upon filtering through a $0.1 \mu \mathrm{m}$ cellulose nitrate filter was a clean solution obtained. Surprisingly, when the same stock solutions were filtered through $0.1 \mu \mathrm{m}$ PVDF filters from Millipore, there was still a large dominant aggregate population, which gave even less peak definition than when the $0.2 \mu \mathrm{m}$ cellulose nitrate filter was used. The $I / k C_{p}$ data from the different filtrations are shown in figure 10. This result shows that extreme care must be taken both in choosing membrane pore diameters and the type of material. 


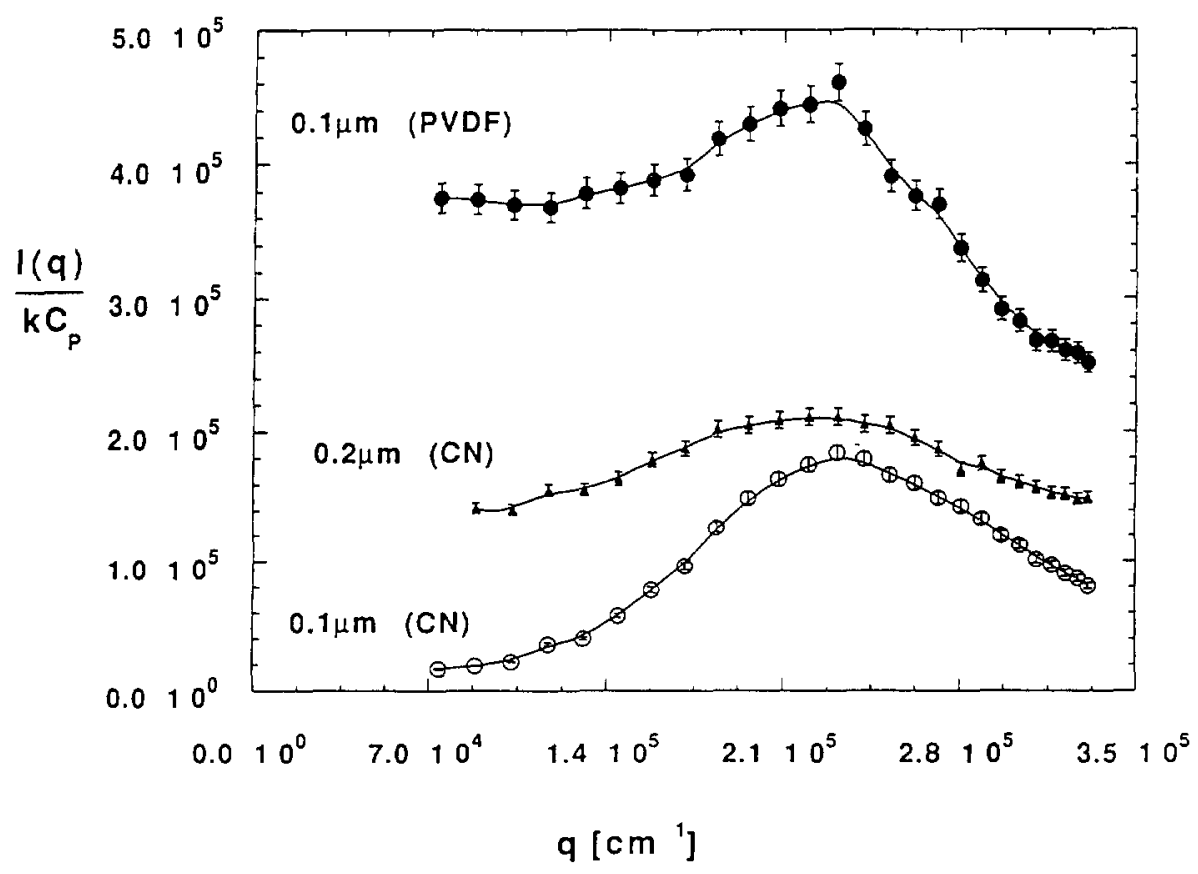

Fig. 10. - Variation of $I / k C_{\mathrm{p}}$ as function of $q$ for $\mathrm{SG}$ filtered through different types of membranes $\left(C_{\mathrm{p}}=3 \times 10^{-5} \mathrm{~g} / \mathrm{cm}^{3}\right)$. Top to bottom; $0.1 \mu \mathrm{m}$ PVFD, $0.2 \mu \mathrm{m}$ cellulose nitrate and $0.1 \mu \mathrm{m}$ cellulose nitrate.

As to the fact that cellulose nitrate cleans up the systems better than PVDF of the same «pore size ", we are not sure exactly why. We now mention, however, that the manufactures quoted « pore size» for most polymeric membranes does not refer to clean, cylindrical round holes in the membranes, actually refers to a sort of average porosity, the «holes » being torturous, complex paths in the membrane. (Exceptions to this are the nucleopore series of membranes, for which the pores are actually close to cylindrical, but which we did not use). Added to this are possible effects of chemical specificity and retention in the membranes.

The possibility that there is only « one characteristic length » in « such complex systems » in understandable in the context of cleaning up the solutions with a particular membrane type and nominal pore size : basically the system is not so complex. In terms of filtrable, large molecules, there are the well-dispersed polymers, which constitute the vast weight percentage of the solute and lead to the interesting physical properties, and a small population of aggregates of entanglements which lead to a «slow» diffusive mode and reduced scattering peak definition, whose average dimensions are larger than the well-dispersed polymers. Using the proper membrane preferentially filters out a large percentage of these large aggregates, allowing the remaining well-dispersed polyelectrolytes to dominate the solution scattering.

Figure 11 a shows autocorrelation functions for SG solutions at $C_{\mathrm{p}}=3 \times 10^{-5} \mathrm{~g} / \mathrm{cm}^{3}$ and $\theta=70^{\circ}$, filtered through $0.1 \mu \mathrm{m}$ cellulose nitrate (curve a) and $0.1 \mu \mathrm{m}$ PVDF filters (curve $b$ ), with monoexponential fits to each shown by the solid lines. As the scattered intensity by polymer changes with the filtration (see Fig. 10) the parameter $\alpha$ (in Eq. (1)) changes. The CN autocorrelation function (curve a), corresponding to the best defined $I / k C_{\mathrm{p}}$ peak in figure 10 (symbol O) is well fit by a single exponential (solid line), whose equivalent $R_{\mathrm{h}}=320 \AA$. The 


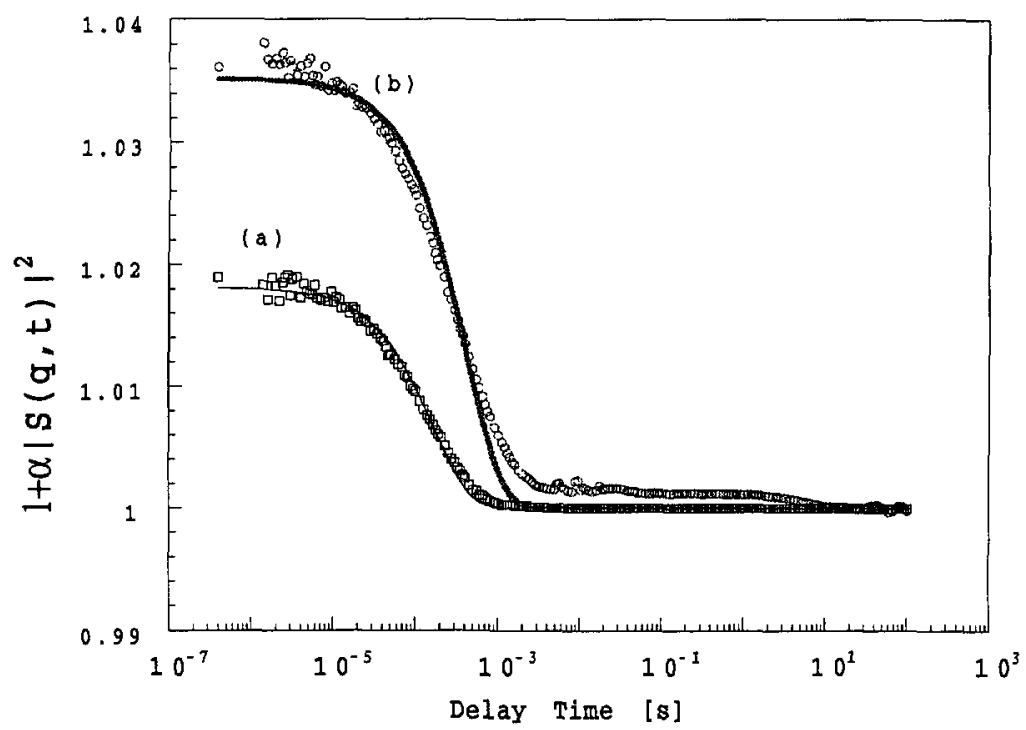

a)

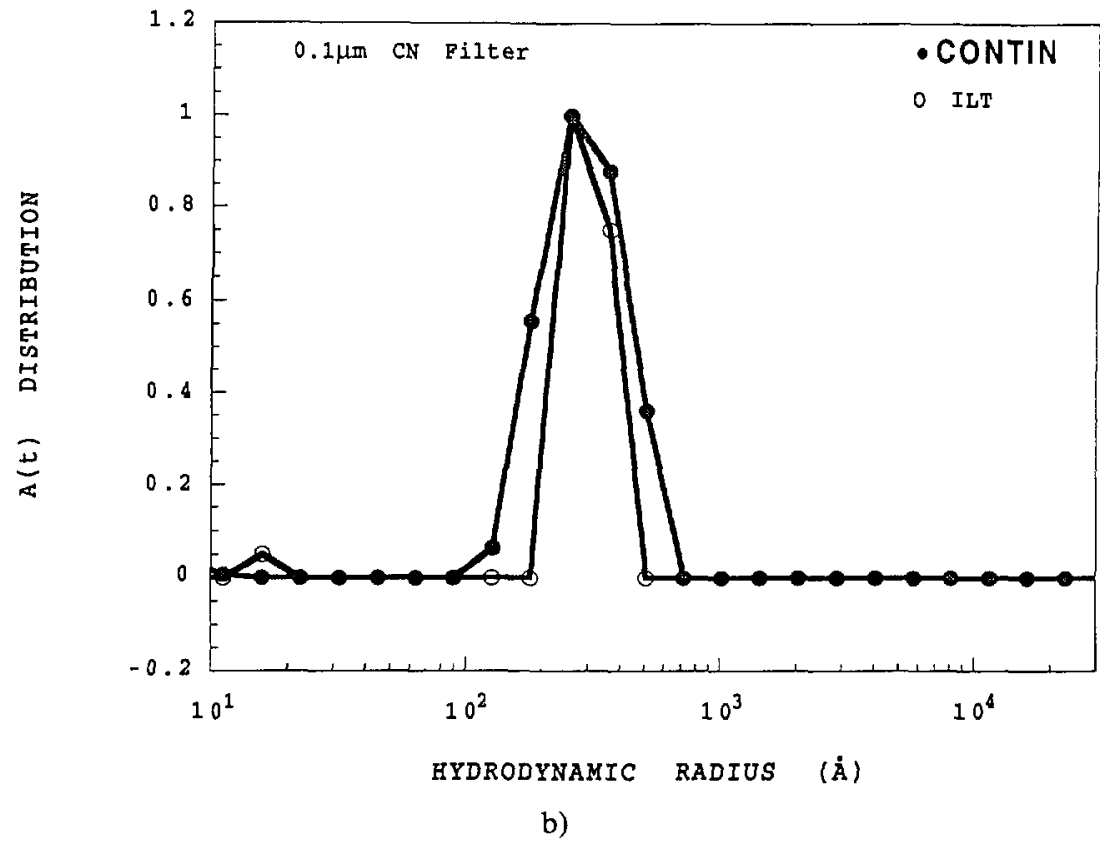

Fig. 11. - a) Typical autocorrelation functions for SG solutions, filtered through $0.1 \mu \mathrm{m}$ cellulose nitrate (curve a) and $0.1 \mu \mathrm{m}$ polyvinylidene fluoride filters (curve b) at $C_{\mathrm{p}}=3.0 \times 10^{-5} \mathrm{~g} / \mathrm{cm}^{3}$ and $\theta=70^{\circ}$. The solid lines represent monoexponential functions. b) CONTIN and ILT analysis of the autocorrelation function for SG at $C_{\mathrm{p}}=3 \times 10^{-5} \mathrm{~g} / \mathrm{cm}^{3}$ and $\theta=70^{\circ}$ filtered through a $0.1 \mu \mathrm{m}$ cellulose nitrate membrane, the filtration method which gives the highest peak contrast and definition. There is a single dominant peak around $R_{\mathrm{h}}=300 \AA$. c) CONTIN and ILT analysis of the autocorrelation function for the same concentration $\left(C_{\mathrm{p}}=3 \times 10^{5} \mathrm{~g} / \mathrm{cm}^{2}\right.$ and $\left.\theta=70^{\circ}\right)$ SG filtered through a $0.1 \mu \mathrm{m}$ polyvinylidene fluoride membrane. In addition to the peak near $R_{\mathrm{h}}=300 \AA$ there is a "slow mode " with $R_{\mathrm{h}}=1000 \AA$, due to the aggregate population. The dashed lines are from figure $11 \mathrm{~b}$, to illustrate that the single mode population for the $0.1 \mu \mathrm{m} \mathrm{CN}$ filtered solution corresponds to the faster mode of the $0.1 \mu \mathrm{m}$ PVDF filtered solution. 


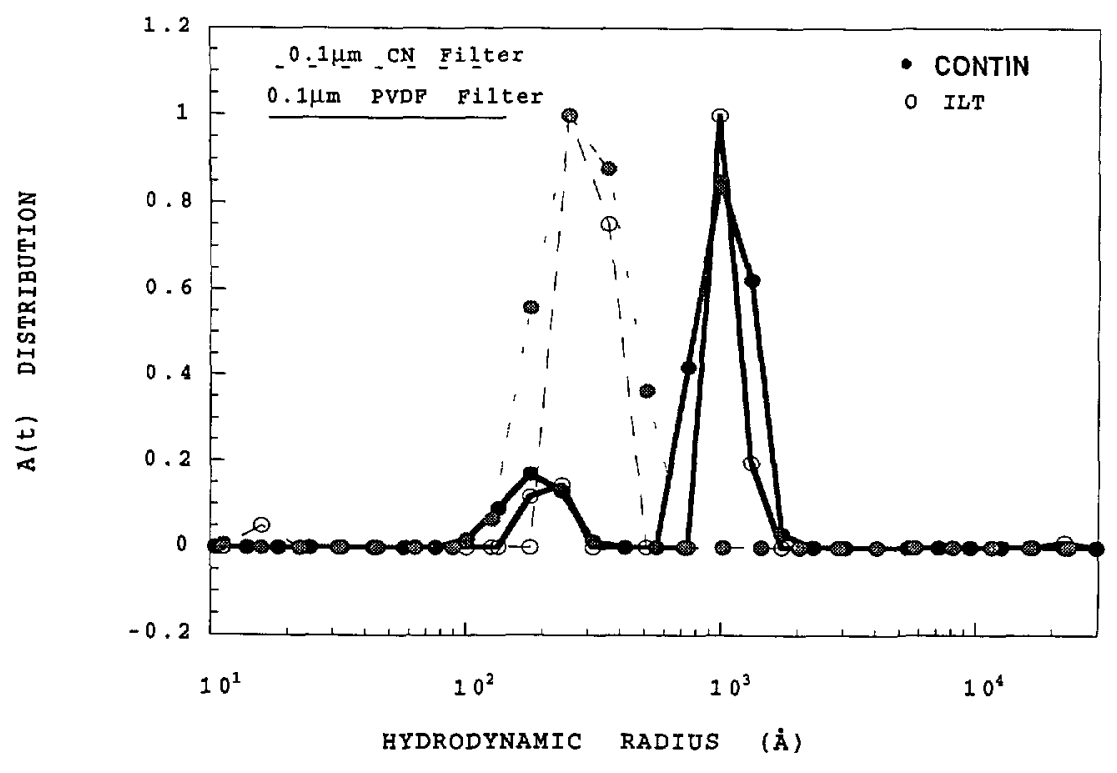

c)

Fig. 11 (contınued).

function for the $0.1 \mu \mathrm{m}$ PVDF filtered solution corresponding to top curve $(\bullet)$ in figure 10 , is not well fit by the single exponential as is readily seen. The same behavior for the other autocorrelation functions and their fits was observed for the angles ranging from $50^{\circ}$ to $150^{\circ}$. One notes that the extremely low values of the intercepts of autocorrelation curves is due to the fact that $I_{\text {poly }} / I_{\text {solv }}$ is extremely low. For the range of concentration studied in this work, the value of the ratio $I_{\text {poly }} / I_{\text {solv }}$ was about 1.6.

As shown in figures $11 \mathrm{~b}$ and $11 \mathrm{c}$ these results are confirmed by CONTIN and ILT analyses of the same autocorrelation functions. The autocorrelation function for a solution filtered with $0.1 \mu \mathrm{m} \mathrm{CN}$ filter, which corresponds to the cleanest solutions with the highest contrast and definition peaks. shows a single main CONTIN/ILT peak at $R_{\mathrm{h}}=300 \AA$ (Fig. $11 \mathrm{~b}$ ). There is no «slow mode», indicating that virtually all the aggregates have been successfully removed by the filtration. The CONTIN/ILT analysis in figure $11 \mathrm{c}$ shows that the solution is full of aggregates, as reflected by the "slow mode " in the distribution function $A(\tau)$, corresponding to the dominant peak with $R_{\mathrm{h}}$ around $1000 \AA$. The smaller peak is from the non-aggregated $S G$ and has an $R_{\mathrm{h}}$ around 200-300 $\AA$, which is close to the value of the single peak in figure $11 \mathrm{~b}$. It should be noted that multimodal decay functions have commonly been observed for salt-free solutions even at such low concentrations as reported here [Refs. 22, 31 and 33].

Thus, the static peaks are most strong and well-defined when there is no slow mode of diffusion. As seen in figure 10, the existence of the slow mode actually decreases the amplitude and contrast of the static scattering peaks; the static scattering peaks and the slow mode are antagonistic to each other.

3.6 VISCOSITY MEASUREMENTS. - Reduced viscosity $\eta_{\mathrm{r}}$ versus $C_{\mathrm{p}}$ taken on the low shear rate Newtonian plateau for SG in pure water is shown in figure 12.

$\eta_{\mathrm{r}}$ increases monotonically over the studied $C_{\mathrm{p}}$ range for the two curves. Thus, over the regime where the pronounced static and dynamic scattering peaks occur, the reduced viscosity behavior is similar to that of most neutral polymers. Conspicuously absent is any trend towards increasing $\eta_{\mathrm{r}}$ with decreasing $C_{\mathrm{p}}$, as is found for dilution with pure water (or low 


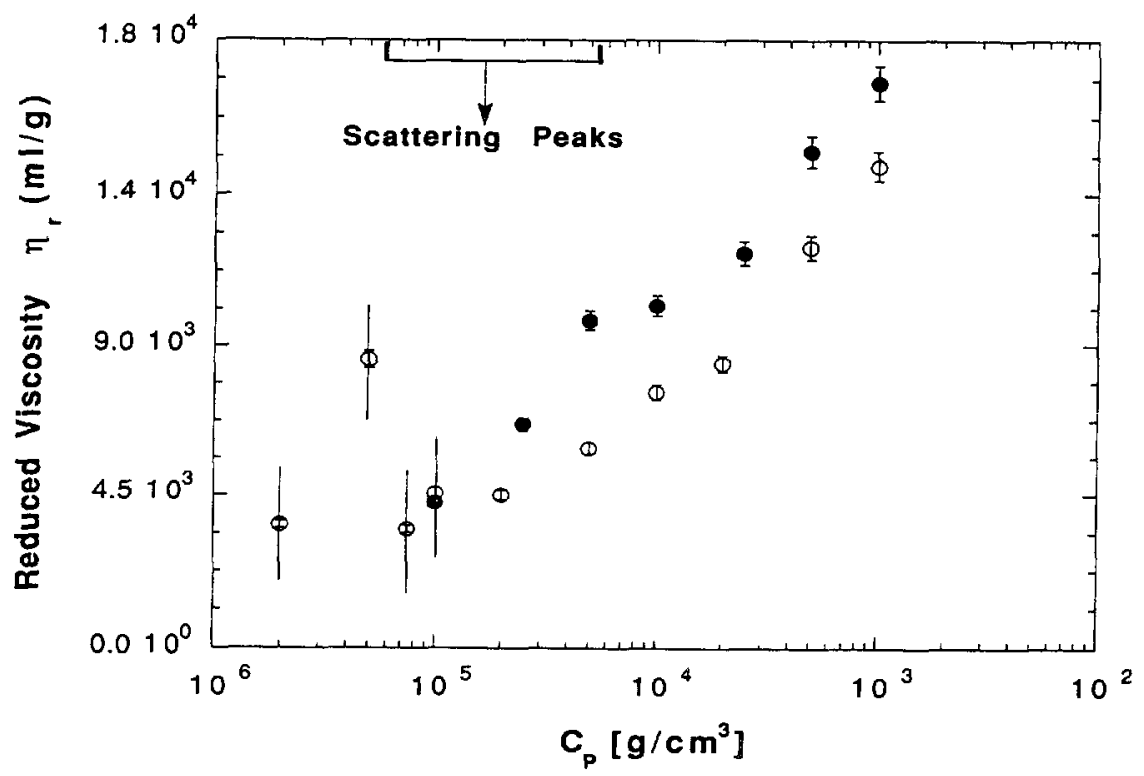

Fig. 12. - Variation of the reduced viscosity $\eta_{\mathrm{r}}$ as function of $C_{\mathrm{p}}$ for $S G$ in pure water. It increases monotonically with $C_{\mathrm{p}}$. The symbol $(O)$ is for the fractionated sonicated sample filtered through $0.1 \mu \mathrm{m} \mathrm{CN}$. The $(\bullet)$ curve is for the same sample filtered through $0.2 \mu \mathrm{m} \mathrm{CN}$.

$C$, solutions) for such semi-flexible polyelectrolytes as polystyrene sulfonate [35], hyaluronic acid [56], gellan, polyvinyl sulphate [57] and charged inextensible latex spheres [58]. Such an effect is traced to the fact that ionic concentration decreases as dilution proceeds. This decrease of ionic strength leads to effects such as: polyelectrolyte expansion, increase in the electrostatic screening length, increase in polyelectrolyte-polyelectrolyte friction, etc. There is no general agreement currently on the exact mechanism of the effects in different situations, although abundant models exist.

Absence of the polyelectrolyte effect - i.e. monotonic increase of $\eta_{\mathrm{r}}$ with $C_{\mathrm{p}}-$ has been reported long time ago for thymonucleic acid TNA [59].

We do not speculate here on whether the monotonic decrease of $\eta_{r}$ with decreasing $C_{\mathrm{p}}$ is due to the more rigid nature of SG compared to these other polyelectrolytes, or possibly to such an effect lying at concentrations lower than the sensitivity of the viscosimeters permitted to measure. In the absence of external salt, the investigated domain of concentration remains in the semi-dilute. The polyelectrolyte effect is usually observed on flexible molecules, short rigid molecules [37] or charged spheres [18]. Moreover, for low charged spheres, it was shown [60] that the behavior mimics that of neutral one (monotonic increase of $\eta_{\mathrm{r}}$ with increasing $C_{\mathrm{p}}$ ).

Succinoglycan has relatively a low charge density $(\lambda=0.45)$ as compared to other polysaccharides. However, the viscosity peak exists for a more flexible polyelectrolyte, hyaluronate $(\lambda=0.7)$ having a charge parameter in the same order as SG. To draw a definitive conclusion on this peculiar behavior, further experiments should be done on different semirigid polyelectolytes having different molecular weigths and different charge densities. What is clear is that the existence of the static and dynamic scattering peaks do not depend on their being any reduced viscosity peak versus $C_{\mathrm{p}}$, nor even a simple increase in $\eta_{\mathrm{r}}$ with decreasing $C_{p}$ in the concentration ranges where the scattering peaks are observed. Furthermore, the fact that the viscosity behavior of the $S G$ when filtered through 0.1 and $0.2 \mu \mathrm{m} \mathrm{CN}$ filters is nearly the same illustrates the fact that the viscosity is closer to a number averaging technique, and is 
not nearly as sensitive to aggregates as light scattering, which combines weight and zaveraging of the particle populations, and is hence extremely sensitive to even small populations of aggregates and other large particles.

\section{Conclusion.}

In this paper we have investigated the structure and dynamic properties of succinoglycan solutions at very low concentrations and ionic strength. Static and dynamic light scattering measurements show the existence of single, pronounced peaks, which are rapidly lost with the addition of salt. While clearly electrostatic in origin, there may yet be some debate about the exact origin of the peaks. The simplest explanation consistent with all the observations is that the electrostatic repulsions at very low $C_{s}$ produce enhanced effective volumes for SG which lead to liquid like correlations. This mechanism has been discussed previously [27. 38]. Liquid-like correlations generally imply strong neighbor-neighbor correlations, with very quickly damped second and more distant neighbor interactions.

It has been demonstrated that the static and dynamic light scattering peaks are unrelated to any slow mode of diffusion (also previously demonstrated for PG [27]), and that in fact such a slow mode, which corresponds to incompletely eliminated aggregates, actually decreases the contrast and definition of the peak when present. Fortunately, these aggregates can be virtually totally eliminated by proper filtration $(0.1 \mu \mathrm{m}$ cellulose nitrate membrane diameter in this case). Such a «slow mode », commonly observed in many polyelectrolyte solutions at low added salt concentration $C_{\mathrm{s}}$, has recently been traced to the presence of aggregates and/or other particles for a variety of polyelectrolyte solutions studied [28].

It has been further demonstrated that the scattering peaks in this case are not related to any peaked behavior of the reduced viscosity $\eta_{\mathrm{r}}$ as a function of $C_{\mathrm{p}}$, nor to any Fuoss-type increase of $\eta_{\mathrm{r}}$ with decreasing $C_{\mathrm{p}}$. The lack of such an effect is perhaps related in this case to the stiffness and to the low charge parameter of this polysaccharide. The effects of charge parameter $\lambda$, preliminary results are presented elsewhere [61].

\section{Acknowledgments.}

R. B. is grateful to Professor M. Benmouna for many helpful discussions. W. R. acknowledges support from NSF (U.S.A.) MCB 9116605, and ELF Aquitaine and the CNRS during his sabbatical as Professeur de l'Académie des Sciences (1992/93).

\section{References}

[1] Katchalsky A., Alexandrowicz Z. and Kedem O., Chemical Physics of Ionic Solutions, Conway and Barradas Eds. (Willey, N.Y., 1976).

[2] de Gennes P. G., Scaling Concepts in Polymer Physics (Cornell University Press, Ithaca. N.Y., 1979), p. $64,65$.

[3] Hayter J., Jannink G., Brochard F. and de Gennes P. G., J Phys. Lett. France 41 (1980) L-451.

[4] de Gennes P. G., Pincus P., Velasco R. M. and Brochard F., I. Phys. France 37 (1976) 1461.

[5] Benmouna M., Weill G., Benoit H. and Akcasu A. Z.. J. Phys. France 43 (1982) 1679.

[6] Odijk T., J. Poly. Sci. Polym. Phys. Ed. 15 (1977) 477.

[7] Skolnick J. and Fixman M., Macromolecules 10 (1977) 9444.

[8] Le Bret M., J. Chem. Phys. 76 (1982) 6273.

[9] Manning G. S., Q. Rev Biophys. 11 (1978) 179.

[10] Hess W. and Klein R., Adv. Polym. Sci. 32 (1982) 173.

[11] Belloni L. and Drifford M., J. Phys. Lett. France 46 (1985) L-1183.

[12] a) Borsali R.. Vilgis T. A. and Benmouna M., Macromolecules 25 (1992) 5313 ;

b) Borsali R. and Rinaudo M., Die Makromol. Theor. Simul. 2 (1993) 179 ;

c) Borsali R., Vilgis T. A. and Benmouna M., Die Makromol. Theor. Simul. 3 (1994) 73.

[13] Odijk T., Macromolecules 12 (1979) 688. 
[14] Witten T. and Pincus P., Europhys. Lett. 3 (1987) 315.

[15] Joanny J. F. and Leibler L., J. Phys. France 51 (1990) 545.

[16] Khokhlov A., J. Phys. A 13 (1980) 979.

[17] Vilgis T. A. and Borsali R., Phys. Rev. A 43 (1991) 6857.

[18] Ise N., Angew. Chem. Int. Ed. Engl. 25 (1986) 323.

[19] Kaji K., Urakawa H., Kanaya T. and Kitamaru R.. J. Phys. France 49 (1988) 993.

[20] Nierlich M. et al., J. Phys. France 40 (1979) 701.

[21] Plestil J. et al., Polymer 27 (1986) 839.

[22] Drifford M. and Dalbiez J. P., J. Phys. Chem. 88 (1984) 5368.

[23] Drifford M. and Dalbiez J. P., Biopolymer 24 (1985) 1501.

[24] Ghosh S., Xiao L., Reed C. E. and Reed W. F., Biopolymers 28 (1990) 1101.

[25] Reed W. F., Ghosh S., Medjhadi G. and François J., Macromolecules 24 (1991) 6189.

[26] Peitzsch R. M., Burt M. and Reed W. F., Macromolecules 25 (1992) 806.

[27] Xiao L. and Reed W. F., J. Chem. Phys. 94 (1991) 4568.

[28] Ghosh S., Peitzsch R. M. and Reed W. F., Biopolymers 32 (1992) 1105.

[29] Lin S. C., Li W. I. and Schurr M. J., Biopolymers 17 (1978) 1041.

[30] Sedlak M. and Amis E. J., J. Chem. Phys. 96 (1992) 817.

[31] Sedlak M. and Amis E. J., J. Chem. Phys. 96 (1992) 826.

[32] Sedlak M., Macromolecules 26 (1993) 1158.

[33] Forster S., Schmidt M. and Antonetti M., Polymer 31 (1990) 781.

[34] Palo D. T. F. and Hermans J. J., Rec. Trav. Chim. (Pays-Bas) 71 (1952) 433.

[35] Cohen J., Priel Z. and Rabin Y., J. Chem. Phys. 88 (1988) 7111.

[36] Ganter J. L., Milas M. and Rinaudo M., Polymer 33 (1992) 113.

[37] Malovikova A., Milas M., Borsali R. and Rinaudo M., Polymer (Preprints) 34 (1993) 1011.

[38] Maier E. E., Krause R., Deggelmann M.. Hagenbtchle M.. Weber R. and Fraden S., Macromolecules 25 (1992) 1125.

[39] Krause R., Maier E. E., Doggelmann M., Angenbuichle M., Schulz S. F. and Weber R., Physica A $160(1989) 135$.

[40] Gravanis G., Milas L., Rinaudo M. and Clarke-Sturman A. J., Int. J. Biol. Macromol. 12 (1990) 195.

[41] Gravanis G., Milas M.. Rinaudo M. and Clarke-Sturman A. J., Int. J. Biol. Macromol. 12 (1990) 201.

[42] Siegert A. J., MIT Rad. Lab Rep (1943) No 465.

[43] Provencher S. W.. Comput. Phys. Commun. 27 (1982) 213 ; lbid 27 (1982) 229.

[44] Tinland B., Mazet J. and Rinaudo M., Makromol. Chem. Rap. Comm. 9 (1988) 69.

[45] Fouissac E., Milas M., Rinaudo M. and Borsali R., Macromolecules 25 (1992) 5613.

[46] Reed C. E. and Reed W. F., J. Chem. Phys. 94 (1991) 8479.

[47] Reed C. E. and Reed W. F., J. Chem Phys. 92 (1990) 6916.

[48] Rinaudo M., Gum Stabilisers for the Food Industry. G. O. Phillıps, D. G. Wedlock, P. A. Williams Eds., vol. 6 (Elsevier, 1992) pp. 51-61.

[49] Ghosh S., Kobal I., Zanette D. and Reed W. F.. Macromolecules (1994) in press.

[50] Wang L. and Bloomfield V., Macromolecules 24 (1991) 5791.

[51] Murray C. A., Sprenger W. O. and Wenk R. A., Phys. Rev. B 42 (1990) 668.

[52] Brown J. C., Pusey P. N., Goodwin J. W., Hewill R. H., J. Phys. A 8 (1975) 664.

[53] Van Winkle D. H. and Murray C. A., Phys. Rev. A 36 (1986) 562.

[54] Tinland B., Maret G. and Rinaudo M., Macromolecules 23 (1990) 596.

[55] Schmitz K., Dynamic Light Scattering by Macromolecules, Chap. 10 (Academic Press, 1990).

[56] Rinaudo M., Milas M., Jouon N. and Borsali R., Polymer 34 (1993) 3710.

[57] Imai N. and Gekka K., Biophys. Chem. 41 (1991) 31.

[58] Yamanaka J., Hashimoto S., Matsuoka H., Kitano H., Ise N., Yamaguchi Y., Saeki S. and Tsubukawa M., Polymer Int. 30 (1993) 233.

[59] Conway B. E. and Butter J. A. V., J. Polym. Sci. 12 (1954) 199.

[60] Yamanaka J., Matsuoka H., Kitano H. and Ise N., J. Colloid. Interf. Sci. 134 (1990) 92.

[61] Borsalı R., Morfin I. and Rinaudo M., Polymer (Preprints) 35 (1994) 136. 